http://jmscr.igmpublication.org/home/

ISSN (e)-2347-176x ISSN (p) 2455-0450

crossref DOI: https://dx.doi.org/10.18535/jmscr/v9i6.37

\author{
Dournal Of Medical Science And Clinical Research \\ IGM Publication \\ An Official Publication of IGM Publication
}

\title{
Adenocystic Carcinoma Palate with Lung Metastasis- A Case Report and Review of Literature
}

\author{
Authors \\ Tarun Kumar ${ }^{1}$, Simrandeep Singh ${ }^{2}$, Hemendra Mishra ${ }^{3^{*}}$, Kapil Suri $^{4}$, Rajiv Sharma \\ ${ }^{1,2}$ Senior Resident, Department of Radiotherapy, VMMC \& Safdarjung Hospital, New Delhi \\ ${ }^{3}$ Junior Resident, Department of Radiotherapy, VMMC \& Safdarjung Hospital, New Delhi \\ ${ }^{4,5}$ Senior Professor, Department of Radiotherapy, VMMC \& Safdarjung Hospital, New Delhi \\ *Corresponding Author \\ Hemendra Mishra \\ Junior Resident, Department of Radiotherapy, VMMC \& Safdarjung Hospital, New Delhi
}

\section{Background}

Tumors of the minor salivary gland origin are uncommon, accounting for about $2 \%$ to $3 \%$ of all malignant neoplasms of the upper aerodigestive tract ${ }^{1}$. Although adenocystic carcinoma (ACC) are common entity among salivary gland tumors but still very less number of cases are reported with lung metastasis. Also ACC is known to show delayed distant metastasis. Due to limited data on ACC with lung metastasis, a lacunae lies in how to manage metachronous lung metastasis after the complete remission of local disease. In this study we report a case report of ACC hard palate with recurrence of lung metastasis after 10 years of locoregional treatment along with review of literature.

\section{Introduction}

The salivary glands consist of the three large, paired major glands - parotid, submandibular, and sublingual and many smaller minor glands located throughout the upper aerodigestive tract. The incidence of Salivary gland malignancies is between 0.4 and 2.6/100,000 per year, with a mean of approximately $1.2 / 100,000 .^{2}$ Tumors of the minor salivary glands tend to occur most often in the hard palate, nasal cavity, and paranasalsinuses areas and thus, the site of origin is related to the density of the minor salivary glands in a particular tissue. ${ }^{3}$ The proportion of malignant tumors increases from parotid (25\%) to submandibular $(43 \%)$ to minor salivary glands $(65 \%){ }^{4}$ The treatment of minor salivary gland tumors varies with location but usually involves an attempt at adequate surgical excision first. Irradiation has been used in surgically inaccessible sites or combined with surgery because of locally aggressive tumor behaviour and the occurrence of incomplete resection. Distant metastases overall are encountered in $3 \%$ to $4 \%$ of patients at presentation and in 33\% after 10 years. They are fairly common with adenoid cystic, salivary duct, squamous cell, and undifferentiated carcinomas; in the case of adenoid cystic carcinomas, they may occur quite late in the course of the disease, without recurrence of the primary tumor. Distant metastases are primarily to lung, bone, and occasionally to the liver. 


\section{Case Report}

A36-year-old woman first presented to the Safdarjung hospital New Delhi in department of radiotherapy in December 2008 with 6-month history of swelling on roof of mouth. She was found to have a $3 \times 2 \mathrm{~cm}$ mass on right side of palate, extended upto the right upper molar, and upto uvula posteriorly. She was treated with a wide local excision followed by histopathology suggestive of T2N0M0 disease. Surgery was followed by post-operative locoregional radiation with 60Gy in 30 fractions with conventional fractionation of 5 fractions a week, to due to positive margin. She was followed up regularly without further therapy.

After around 10 years, due to difficulty in breathing, patient was asked to get CECT thorax and then followed by PET CT. It showed a heterogeneously enhancing mass with spiculated margins of size $3.3 \times 3.6 \times 2.5 \mathrm{~cm}$ in right upper lobe of lung with SUV max 8.5. Biopsy of lung mass showed metastatic Adenocystic carcinoma. Patient was discussed in tumor board and started with chemotherapy with inj. Adriamycin $75 \mathrm{mg}$, inj. Cyclophosphamide 750mg and inj. Cisplatin 150 mg iv 3 weekly. After 6 cycles of chemotherapy, patient had subjective response with no signs of breathing difficulty and in PET-CT the size of the

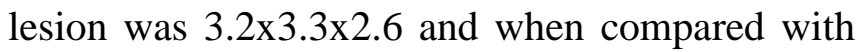
previous PET-CT, it suggested stable disease. Case was again discussed in tumor board. Patient was offered with option of metastasectomy. Patient was not willing for surgery, and was asymptomatic. Thus patient was decided to be kept on close follow up.

\section{Discussion}

Adenocystic carcinoma of minor glands with metastasis in lungs is over all a rare case in numbers. In a study in 2019 by Jungirl Seok et al showed that lung metastasis are $45 \%$ in ACC Head and neck (median time to lung metastasis 2.67 years) with more propensity in Sub lingual and minor salivary glands (70\% vs 29\%) compared to parotid and submandibular gland.
Also they concluded that chances are more if primary tumor size is more than $2.5 \mathrm{~cm}(70 \% \mathrm{vs}$ $23 \%$ ) compared to tumor less than $2.5 \mathrm{~cm} .{ }^{6}$ Due to rarity of the scenario there is a lacunae in consensus on management of lung metastasis. Different groups all over the world have tried different approaches like surgical resection, chemotherapy, targeted therapy and radiotherapy, for the management of distant lung metastasis.

\section{Surgical Resection}

The role of surgery in the treatment of pulmonary metastases is well-defined in a large variety of cancers, but few reports have examined the outcomes in patients undergoing lung resection for metastatic ACC.

In 2016 Girelli et al. in milan Italy published a study of 109 cases which showed that complete resection of lung metastasis in ACC have 5years OS $70 \%$ and 10 years OS $46 \%$ compared to incomplete resection (50\% and $0 \%$ respectively). Also their data suggest that OS for patients with disease free interval (DFI) less than 36 months was $47.7 \%$ at 5 years and $21.9 \%$ at 10 years compared to patients with DFI more than 36 months, $76.5 \%$ and $51.1 \%$ respectively. And thus concluding Lung metastasectomy should be considered as a therapeutic option to achieve local control of disease when: (1) complete surgical resection is feasible and (2) the time to pulmonary relapse after primary tumor treatment is greater than 36 months. ${ }^{7}$

In 2008 Bobbio et al. published a data of 20 patients who were diagnosed with lung metastasis in head and neck ACC. It showed that post treatment of primary mean DFS was 36 months and median survival after lung metastasectomy in 11 patients was 72 months. Median survival of patients diagnosed as lung metastasis but didn't underwent resection was 62 months with Kaplan mier curve being statistically non significant, for which small sample size may be a reason. ${ }^{8}$ In a review of lung metastasectomy for ACC, Locati et al. retrospectively analysed data from 20 patients with ACC of salivary gland origin. 
Eleven patients had no residual disease (R0) after metastasectomy and 9 patients had residual disease (R2). Of the 11 disease free patients, 4 were alive after 4 years after surgery without evidence of disease. Of the 9 patients with macroscopic residual disease, 4 were alive after 4 years, including 2 who had experienced a local recurrence at the primary tumorsite. Median survival after metastasectomy in the RO and R2 groups was 78 and 52 months, respectively. Subgroup analysis revealed that a disease-free interval of more than 36 months and a low tumor burden defined a group of patients who had a better prognosis after metastasectomy. ${ }^{9}$

So according to authors surgery is a viable option in case lung metastasis on head and neck ACC. Most likely to be benefitted patients are the one who had more than 36 years of DFS after treatment of primary tumor and complete resection of metastasis with adequate postoperative reserve of lung tissue.

\section{Systemic Therapy}

The primary use of chemotherapy or systemic therapy is for patients with recurrent or metastatic disease for whom local therapy (surgery or radiotherapy) is not possible. Rigorous testing or standardization of systemic therapies in metastatic or recurrent salivary gland carcinomas is extremely difficult due to the rarity and heterogeneity of these tumors. Multiple single agents like paclitaxel, gemcitabine, mitoxantrone and platins have been tried in metastatic ACC but none had showed any clinically and statistically significant improvement in survival.

In a study by Hill $M$, patients taken for palliative chemotherapy of metastatic, symptomatic, histologically confirmed adenoid cystic carcinoma were considered for this study. The drugs were administered as follows: cisplatin $80 \mathrm{mg} / \mathrm{m}^{2}$ with divided doses and 5-FU 4days schedule of 1 $\mathrm{g} / \mathrm{m}^{2} /$ day. There were no objective responses of $>50 \%$ reduction in tumour size. The symptomatic response rate, however, was $64 \%$, with manageable toxicity. The median time to tumour progression was 9 months and median survival was 12 months. The cisplatin/5-FU regimen would appear to produce a low rate of objective response but useful palliative benefits in advanced symptomatic adenoid cystic carcinoma. ${ }^{10}$

Triplet therapy with platinum was reported in a small study by Ross et al. investigating the combination of cisplatin/carboplatin, epirubicin, and 5-flurouracil (5-FU) in eight patients with ACC, seven as first-line therapy. Despite the addition of a third cytotoxic agent, the ORR was only $12 \%$, further supporting the notion that triplet cytotoxic regimens have no clinical benefit for metastatic salivary cancers. Even though the ORR was modest, the median OS was still 27 months underscoring the of tenindolent clinical behaviour of metastatic ACC and the dual challenges of studying systemic therapy and having transient disease response translate into a meaningful survival advantage. ${ }^{11}$

The most studied chemotherapeutic regimen was the historic salivary cancer regimen cyclophosphamide, doxorubicin, and cisplatin. Cisplatin-anthracycline-based combination regimens also have activity in ACC. For example, in five studies of the regimen cisplatindoxorubicin-cyclophosphamide (CAP) with or without fluorouracil, objective responses were observed in 12 of 43 patients (28\%; 95\% CI, $17 \%$ to $43 \%$ ). Overall, the data are insufficient to conclude that combination therapy is superior to single-agent therapy, and the likelihood of additional toxicity of a combination regimen needs to be considered. ${ }^{12}$

Epidermal growth factor receptor (EGFR) is highly expressed in adenoid cystic carcinoma (ACC). The efficacy and toxicity of cetuximab with concomitant platinum-based chemotherapy in patients with metastatic ACC, was evaluated in a study in 2013. In metastatic cases, cetuximab loading dose $\left(400 \mathrm{mg} / \mathrm{m}^{2}\right)$ was followed by $250 \mathrm{mg} / \mathrm{m}^{2}$ per week concomitant cisplatin and 5fluorouracil $\left(4 \times 1000 \mathrm{mg} / \mathrm{m}^{2}\right)$. With median follow-up of 72 months, the median PFS and OS were 13 and 24 months, respectively. Objective 
response rate was $>40 \%$ with Skin rash, mucositis and vomiting were the most frequent grade 3/4 adverse events. $^{13}$

A phase II study of gefitinib in advanced, progressive salivary malignancies has been reported in abstract form, Of the 29 patients enrolled, 19 had ACC. No major objective responses were observed; 10 (53\%) of 19 had disease stabilization, which has been maintained for at least 16 weeks in five patients. ${ }^{14}$

Patients with adenoid cystic carcinoma of the salivary glands show over-expression of KIT in a high proportion of cases. Options for systemic treatment are limited in locally advanced and metastatic disease. In 2011 in a study by Ghosal et al. explored the efficacy of imatinib and cisplatin combined in this group of patients. A phase II trial was conducted on 28 ACC of salivary glands patients. Those with over-expression of KIT were treated with single agent imatinib $800 \mathrm{mg}$ daily for two months, followed by a combination of imatinib $400 \mathrm{mg}$ daily and cisplatin $80 \mathrm{mg} / \mathrm{m} 2$ at four-weekly intervals for six cycles. This was followed by maintenance single agent imatinib $400 \mathrm{mg}$ daily until the disease progressed. Imaging showed partial response in three of 28 patients, and five patients showed a response on FDG-PET. In addition, 19 patients had useful stabilisation of disease. The median time to progression and overall survival was 15 months (range 1-43) and 35 months (range 1-75), respectively. The combination of imatinib and cisplatin was reasonably well tolerated. This combination may provide stabilisation in locally advanced and metastatic adenoid cystic carcinoma of the salivary glands. ${ }^{15}$

Salivary gland carcinomas are notoriously resistant to therapy and no standard of care exists. Due to the rarity of these malignancies, various histologic, and wide ranging clinical behaviour, it has been difficult to standardize systemic therapy. Chintakuntlawar et al have reviewed clinical prospective studies in the last 15 years with salivary gland malignancies involving cytotoxic chemotherapy and biologic agents including targeted therapies such as anti-HER-2, anti-EGFR therapies, and therapies directed at c-kit. Although the results of most trials are modest at best, there has been an increase in studies for salivary cancer in recent years and there are several promising treatment approaches in evolution. ${ }^{16}$

According to authors Platinum-based chemotherapy with or without EGFR targeted therapy, if chemotherapy is given, remains the best option but falls short of being efficacious enough to be considered standard of care.

\section{Radiation Therapy}

In 2017 in a study by ricco et al 557 patients were studied who received SBRT to lung metastasis. Median dose was $50 \mathrm{~Gy}$ delivered in 3 fractions with a median BED of $100 \mathrm{~Gy}_{10}$. Median OS for the entire group was 26 months, with actuarial 1, 3 and 5years OS 74.1\%, 33.3, and $21.8 \%$, respectively. Patients with head and neck and breast cancers had longer median OS of 37 and 32 months respectively, compared to colorectal (30 months) and lung (26 months) which corresponded to 3-years actuarial OS of 51.8 and $47.9 \%$ for head and neck and breast respectively, compared to $35.8 \%$ for colorectal and $31.2 \%$ for lung. The median LC for all patients was 53 months, with actuarial 1, 3 and 5years LC rates of $80.4,58.9$, and $46.3 \%$ respectively. There was no difference in LC by primary histologic type $(p=0.49)$. Improved LC was observed for lung metastases that received SBRT doses of BED $\geq 100 \mathrm{~Gy}_{10}$ with 3 -years LC rate of $77.1 \%$ compared to $45 \%$ for lung metastases treated with $\operatorname{BED}<1_{100 y_{10}}(p=0.01)$. Smaller tumor volumes $(<11 \mathrm{cc}$ ) had improved LC compared to tumor volumes $>11 \mathrm{cc} .(p=0.005)$ Two-year LC rates for tumor volumes $<11 \mathrm{cc}, 11-27 \mathrm{cc}$ and $>27 \mathrm{cc}$ were 72.9, 64.2 and $45.6 \%$, respectively. This correlated with improved OS with 2-year OS rates of $62.4,60.9$ and $46.2 \%$ for tumor volumes $<11 \mathrm{cc}, 11-27 \mathrm{cc}$ and $>27 \mathrm{cc}$, respectively $(p=0.0023)$. In a subset of patients who received $\mathrm{BED} \geq 100 \mathrm{~Gy}_{10}$, 2 years $\mathrm{LC}$ rates for tumor volumes $<11 \mathrm{cc}, 11-27 \mathrm{cc}$ and $>27 \mathrm{cc}$ were 
$82.8,58.9$ and $68.6 \%$, respectively $(p=0.0244)$, and 2-year OS rates were 66.0, 58.8 and $28.5 \%$, respectively $(p=0.0081)$. but the problem in this study was it had only $11,4 \%$ of head and neck patients and very less info is provided about the histology of the disease. ${ }^{17}$

In 2016 The Surveillance, Epidemiology, and End Results database (SEER) and the National Cancer Data Base (NCDB) were queried for patients with T0-4, N0-3, or M1 salivary ACC by McDermott et al. Two hundred seventy-seven patients from the SEER and 158 patients from the NCDB were identified. From the SEER, 221 underwent LT (70 RT, 66 surgery, 85 both surgery and RT), and 56 had NLT. Patients who received any LT had non significant increased median OS (30 months) compared to NLT (16 months) (hazard ratio [HR] $0.71,95 \%$ CI $0.50-1.02, P=.063)$. When subdividing by treatment type, the median OS difference became significant with NLT at 16 months, RT at 17 months (HR 1.21, 95\% CI 0.811.84 ), surgery at 50 months (HR $0.58,95 \% \mathrm{CI}$ $0.38-0.89$ ), and surgery plus RT at 50 months (HR $0.56,95 \%$ CI 0.37-0.84) $(P<.001)$.

From the NCDB, 105 patients underwent LT (10 RT, 63 surgery, 32 surgery and RT) and 53 had NLT. Patients receiving LT had a significantly longer median OS (26.2 months) compared to NLT (14.5 months) (HR 0.56, 95\% CI 0.39$0.82, P=.003)$. The differences between treatment subtypes were also significant with NLT at 14.5 months, RT at 23.8 months (HR 0.92, 95\% CI $0.42-1.79$ ), surgery at 20.7 months (HR $0.53,95 \%$ CI 0.35-0.81), and surgery plus RT at 28.8 months $\left(\right.$ HR $0.52,95 \%$ CI 0.31-0.86) $(P=.013) .{ }^{18}$

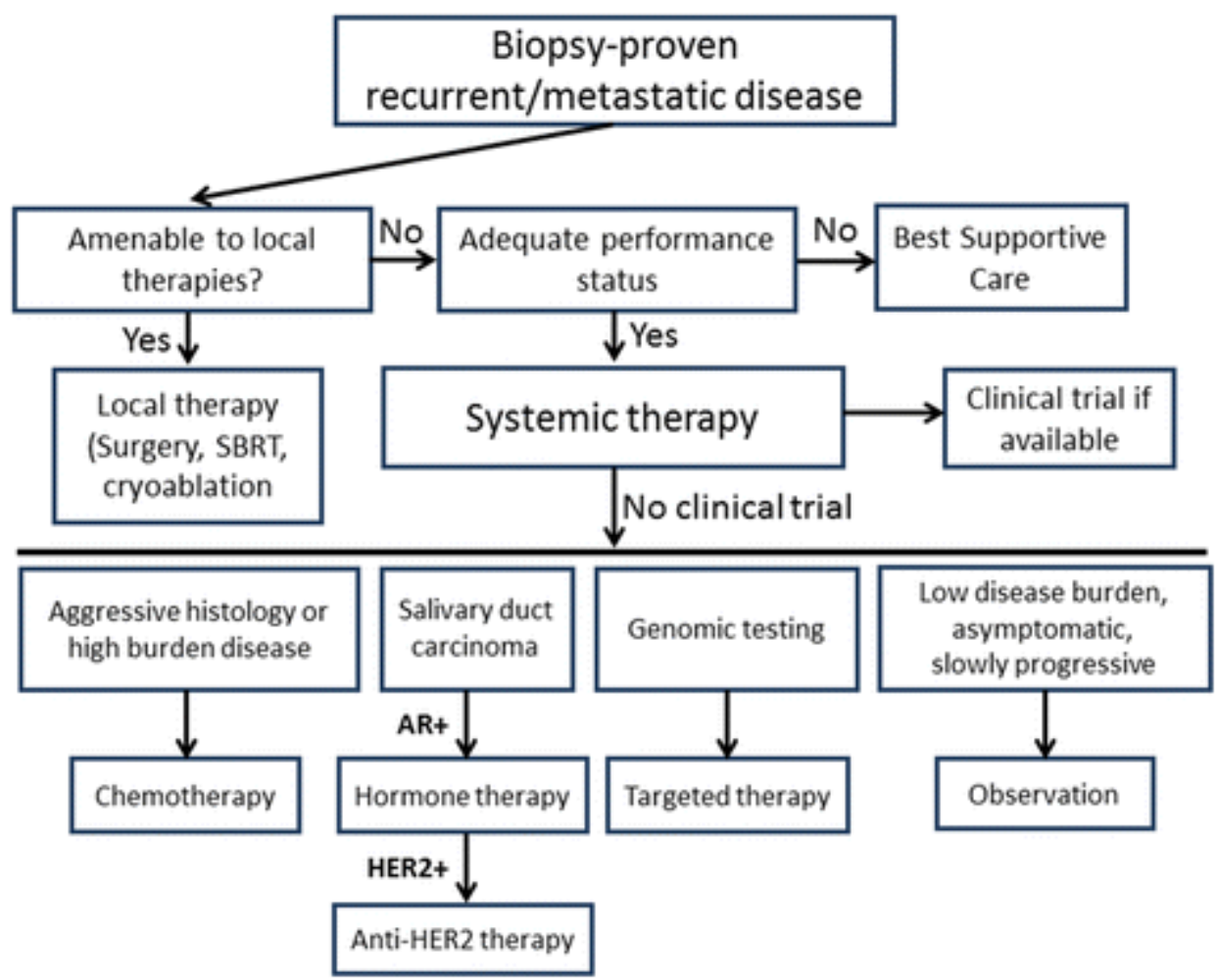

Figure 1.Proposed Plan of Management

Single site of symptomatic metastasis may be more appropriately treated with surgery or radiation. Systemic therapy should be reserved for those with symptoms and/or rapidly progressive disease. With the lack of high quality evidence to guide treatment and the heterogeneity of the tumors, a standard of care treatment approach to patients with recurrent or metastatic salivary is difficult to devise but with the available data we generally follow the approach outlined in Fig. 1. If the patient has a low disease burden with isolated or oligometastatic disease that is amenable to local 
therapies, then we typically recommend local therapy with stereotactic body radiation therapy or surgery. If a patient does not have an adequate performance status, then best supportive care is appropriate. In a fit patient the need to treat is balanced with the risks of systemic therapy. If therapy is required due to disease burden, symptoms, or an aggressive clinical disease, or if the patient desires treatment (after discussion of risks and benefits), we consider systemic treatment with either standard chemotherapy, targeted therapy.

\section{Conclusion}

For those patients with no symptoms and indolent disease, and particularly those with only pulmonary metastases, watchful waiting is often the most appropriate recommendation.

\section{References}

1. Devitapg 1077

2. Bjørndal K, Krogdahl A, Therkildsen MH, et al. Salivary gland carcinoma in Denmark 1990-2005: a national study of incidence, site and histology.Results of the Danish Head and Neck Cancer Group (DAHANCA)Oral Oncol 2011;47:677682.

3. Devitapg 1077

4. Spiro R. Salivary neoplasms: overview of a 35-year experience with 2,807 patients. Head Neck Surg 1986;8:177-184.

5. Terhaard $\mathrm{CH}$, Lubsen $\mathrm{H}$, Van der Tweel I, Hilgers FJ, Eijkenboom WM, Marres HA, Tjho-Heslinga RE, de Jong JM, Roodenburg JL; Dutch Head and Neck Oncology Cooperative Group. Salivary gland carcinoma: independent prognostic factors for locoregional control, distant metastases, and overall survival: results of theDutch head and neck oncology cooperative group. Head Neck. 2004 Aug;26(8):681-92;discussion 692-3.
6. Seok J, Lee DY, Kim WS, et al. Lung metastasis in adenoid cystic carcinoma of the head and neck. Head \& Neck. 2019;41.

7. Girelli L, Locati L, Galeone C, Scanagatta P, Duranti L, Licitra L, Pastorino U. Lung metastasectomy in adenoid cystic cancer: Is it worth it? Oral Oncol. 2017 Feb;65:114-118.

8. Bobbio et al. / European Journal of Cardiothoracic Surgery 33 (2008) 790-793.

9. LocatiLD,Guzzo M, bossip, et al. Lungmetastasectomy in adenoid cystic carcinoma (ACC) of salivary gland. Oral Onco!2005;41(9): 890-4.

10. Hill ME, Constenla DO, A'Hern RP, et al. Cisplatin and 5-fl uorouracil for symptom control in advanced salivary adenoid cystic carcinoma. Oral Oncol 1997; 33: 275-78.

11. Epirubicin, Cisplatin and Protracted Venous Infusion 5-Fluorouracil Chemotherapy for Advanced Salivary Adenoid Cystic Carcinoma Ross, P.J. et al.Clinical Oncology, Volume 21, Issue 4, $311-314$.

12. Laurie SA, Licitra L. Systemic therapy in the palliative management of advanced salivary gland cancers. J Clin Oncol. 2006;24(17):2673-8.

13. Hitre E, Budai B, Takacsi-Nagy Z, Rubovszky G, Toth E, Remenar E, et al. Cetuximab and platinum-based chemoradio- or chemotherapy of patients with epidermal growth factor receptor expressing adenoid cystic carcinoma: a phase II trial. $\mathrm{Br} \mathrm{J}$ Cancer. 2013; 109:1117-22.

14. Glisson B, Blumenschein G, Francisco M, et al: Phase II trial of gefitinib in patients with incurable salivary gland cancer. J Clin Oncol 23:580S, 2005.

15. Phase II study of cisplatin and imatinib in advanced salivary adenoid cystic carcinoma Ghosal, Niladri et al.British Journal of Oral and Maxillofacial Surgery, Volume 49, Issue 7, 510 - 515. 
16. Chintakuntlawar, A.V., Okuno, S.H. \& Price, K.A. Systemic therapy for recurrent or metastatic salivary gland malignancies. Cancers Head Neck 1, 11 (2016).

17. Ricco, A., Davis, J., Rate, W. Et al. Lung metastases treated with stereotactic body radiotherapy: the rssearch ${ }^{\circledR}$ patient Registry's experience. Radiat Oncol 12, 35 (2017).

18. Local Therapy for Metastatic Salivary Adenoid Cystic Carcinoma: A Surveillance, Epidemiology, and End Results and National Cancer Data Base Analysis medermott, J. Et al. International Journal of Radiation Oncology • Biology • Physics, Volume 94, Issue 4, 953. 